\title{
Context-Aware User Modeling and Semantic Interoperability in Smart Home Environments
}

\author{
Giorgos Siolas, George Caridakis, Phivos Mylonas, Stefanos Kollias, and Andreas Stafylopatis \\ Intelligent systems, Content and Interaction Laboratory \\ School of Electrical and Computer Engineering \\ National Technical University of Athens \\ Iroon Polytexneiou 9, 15780 Zografou, Greece \\ Email: \{gsiolas, gcari, fmylonas\}@image.ntua.gr, \{stefanos, andreas\}@cs.ntua.gr
}

\begin{abstract}
Current paper investigates how user modeling, context awareness and content adaptation in Smart Home environments can be handled formally in order to capture the semantics that emerge from a newly introduced user experience: SandS is in fact a complete ecosystem of users within a social network, creating and exchanging content in the form of so-called recipes and developing a collective intelligence which adapts its operation through appropriately feedback provided by the user. We will approach SandS from the user's perspective and illustrate how users and their relationships can be modeled through a number of fuzzy stereotypical profiles. Additionally, context modeling in pervasive computing systems and especially in the Smart Home paradigm will be examined through appropriate representation of context cues in the overall interaction. Finally we will investigate how users and system services although using languages of different semantic expressiveness can inter-operate successfully thanks to appropriate knowledge-based expert mappings.
\end{abstract}

Keywords-User Modeling, Context Awareness, Personalization, Smart Homes, Social Network, Content adaptation, Semantic Interoperability.

\section{INTRODUCTION}

Although in their initial definition and development stages pervasive computing practices did not necessarily relied on the use of Internet, current trends show the emergence of many convergence points with the Internet of Things (IoT) paradigm where objects are identified as Internet resources and can be accessed and utilized as such. Smart Homes that adhere to the IoT approach emerge the users in environments where data is continuously produced by appliances, sensors and humans while data processing, knowledge assessment and decision making can be performed remotely. Following a pragmatic approach, the FP7 European Project and FIRE framework "Social \& Smart" (SandS) [1] aims to highlight the potential of IoT technologies in a concrete user-centric framework. The aim is for the user (eahooker in SandS) to collectively, via the SNS (Social Network Service), and intelligently, via the adaptive social network intelligence, interface and finally control his household appliances. The overall data transfer is orchestrated through a domestic infrastructure. The central role of the user is reflected on all aspects of the ecosystem, from the family of Things which is socially governed to the household appliances that affect our everyday life to the parameters of the domestic and network intelligence. This entire procedure is devised so as to optimally carry out ordinary housekeeping tasks with a minimal low level intervention from the part of the user while preserving acquired knowledge through an interoperable manner.

The main content medium inside Sands are "recipes", messages containing a series of instructions in near natural, user friendly, language for controlling the appliances in order to perform a specific task. Recipes are exchanged between the users of the social network and are in turn adapted to each household. Eahookers are considered definitely as pro-active users: they participate actively in content creation, modification and sharing but also produce additional qualitative content through feedback and recommendations about the recipes effectiveness. Moreover, by giving the means to the eahooker to intelligently control his domestic appliances and by placing him inside the ESN (Eahookers Social Network), SandS follows clearly a human and user centric approach. More precisely, User Modeling (UM) and adaptation emerges as an important research direction inside the project. More precisely, UM not in a general sense, but relatively to the users activity inside the ESN and with respect to the task on hand, the efficient orchestration of his household appliances (context). We are considering in particular a context-aware UM of eahookers, that is taking into account all the contextual information that could characterize the situation and condition of the systems entities. In SandS case this could be context information about the eahooker (distance to his house, communication device used, time of the day, weather, etc.), usage information (recipes used, feedback provided by user, frequency of use,...), information about the homes (geolocalisation, proximity to other homes, surface area, number of rooms, etc.), about the appliances (location inside house, energy consumption levels etc) and information specific to the social network itself (friendship statements, content exchanged between users, graph structure, communities formation, etc.). Eahookers will be recorded and Computational Intelligence algorithms will extract knowledge about groups of similar users and construct for these groups stereotypical users (Personas).

Current paper investigates how each individual eahooker could be modeled with a simple user model, consisting of a fuzzy representation of extracted Personas. The main goal 
is to capturer the semantics of the relationships between the user and the various entities of the ecosystem (recipes, other users, houses, appliances and manufacturers). Finally, an important interoperability issue will be tackled, namely how to bridge the gap between the expressively rich natural language vocabulary used in the recipes and the low level machinereadable instructions with very precise and restricted semantic content.

\section{USER AND CONTEXT MODELING}

A user model [2] is a computational representation of the information existent in a user's cognitive system, along with the processes acting on this information to produce an observable behavior. User stereotypes or personas is a quite common approach in UM due to its correlation with the actors and roles used in software engineering systems, its flexibility, extensibility, reusability and applicability. A persona is an archetypal user derived from specific profile data to create a representative user containing general characteristics about the users and user groups and is used as a powerful complement to other usability methods. The use of personas is an increasingly popular way to customize, incorporate and share the research about users [3]. The personas technique fulfills the need of mapping and grouping a huge number of users based on the profile data, aims and behavior which can be collected both during design and run time, users and usage design respectively.

Recently, the emergence of ubiquitous or pervasive computing technologies that offer "anytime, anywhere, anyone" computing by decoupling users from devices have introduced the challenge of context-aware user modeling. Context is information used to characterize the situation of an entity. An entity is a person, place, or object that is considered relevant to the interaction between a user and an application, including location, time, activities, and the preferences of each entity. A user model is context-aware if it can express aspects of the user's contextual information and subsequently help the system adapt its functionality to the context of use. Many aspects of contextual information used in modeling are discussed in [4] and [5] however a few authors have addressed utilizing the cognitive elements of a user's context and the semantics of the relations between the user and the system's entities.

\section{FUZZY PERSONAS}

Motivation and development of our knowledge modeling notion and methods are grounded on a set of problems, assumptions, views and design decisions, which are stated next. We consider following settings: A set of users $\mathcal{U}$ interact with information objects, typically (though not mandatorily) containing a fair amount of unstructured or semi-structured content, e.g. text and/or multimedia objects and/or documents. The information objects are annotated with metadata, consisting of concepts, properties and values defined according to a domain ontology $\mathcal{O}$, and stored in a knowledge base (KB). At this point it should be made clear that the latter constitutes a clear assumption of this work and ontology matching or semantic similarity issues are not tackled herein.
Following the above common view, we define $P$ as a set of meanings that can be found or referred to in items. Beyond raw keywords and multimedia descriptors, which are commonly used as semantic representation bricks for user needs, ontologies are being investigated in the field as enablers of qualitatively higher expressivity and precision in such descriptions [6], [7], [8], [9]. In our approach, $P$ is described as a set of semantic entities that the user has interest for to varying degrees. This provides a fairly precise, expressive, and unified representational grounding, in which both user interests and content meaning are represented in the same space, in which they can be conveniently compared [10].

Efficient user model representation formalism using ontologies ([11], [12]), present a number of advantages. In the context of this work, ontologies are suitable for expressing user modeling semantics in a formal, machine-processable representation. As an ontology is considered to be " a formal specification of a shared understanding of a domain', this formal specification is usually carried out using a subclass hierarchy with relationships among classes, where one can define complex class descriptions (e.g. in Description Logics (DLs) [11] or Web Ontology Language (OWL) in [13]).

\section{A. Mathematical background}

Given a universe $\mathcal{V}$ of users $\mathcal{U}$, a crisp (i.e., non fuzzy) set $S$ of concepts on $\mathcal{V}$ is described by a membership function $\mu_{S}: \mathcal{V} \rightarrow\{0,1\}$. The crisp set $S$ may be defined as $S=$ $\left\{s_{i}\right\}, i=1, \ldots, N$. A fuzzy set $F$ on $S$ may be described by a membership function $\mu_{F}: S \rightarrow[0,1]$. We may describe the fuzzy set $F$ using the well-known sum notation for fuzzy sets [14] as:

$$
F=\sum_{i} s_{i} / w_{i}=\left\{s_{1} / w_{1}, s_{2} / w_{2}, \ldots, s_{n} / w_{n}\right\}
$$

where:

- $i \in N_{n}, n=|S|$ is the cardinality of the crisp set $S$,

- $w_{i}=\mu_{F}\left(s_{i}\right)$ or, more simply $w_{i}=F\left(s_{i}\right)$, is the membership degree of concept $s_{i} \in S$.

Consequently, equation (1) for a concept $s \in S$ can be written equivalently as:

$$
F=\sum_{s \in S} s / \mu_{F}(s)=\sum_{s \in S} s / F(s)
$$

Let now $R$ be the crisp set of fuzzy relations defined as:

$$
R=\left\{R_{i}\right\}, R_{i}: S \times S \rightarrow[0,1], \quad i=1, . ., M
$$

Then, in compliance to the notion introduced in [15], the proposed fuzzy ontology will contain concepts and relations and may be formalized as follows:

$$
\mathcal{O}=\{S, R\}
$$

In equation (4), $\mathcal{O}$ is a fuzzy ontology, $S$ is the crisp set of concepts described by the ontology and $R$ is the crisp set of fuzzy semantic relations amongst these concepts.

Given the set of all fuzzy sets on $S, \mathcal{F}_{S}$, then $F \in \mathcal{F}_{S}$. Let $\mathcal{U}$ be the set of all users $\widehat{u}$ in our framework, i.e. a user $\widehat{u} \in \mathcal{U}$. 
Let $\mathcal{P}$ be the set of all user meanings and $\mathcal{P}_{\mathcal{O}}$ be the set of all user meanings on $\mathcal{O}$. Then $\mathcal{P}_{\mathcal{O}} \subset \mathcal{F}_{S}$ and $\mathcal{P}_{\mathcal{O}}=\mathcal{F}_{Z} \subset \mathcal{F}_{S}$, whereas $P_{\widehat{u}} \in \mathcal{P}_{\mathcal{O}}$ depicts a specific user meaning.

\section{B. Personas fuzzy relations}

In order to define, extract and use both a set of concepts, we rely on the semantics of their fuzzy semantic relations. As discussed in the previous subsection, a fuzzy binary relation on $S$ is defined as a function $R_{i}: S \times S \rightarrow[0,1], i=1, . ., M$. The inverse relation of relation $R_{i}(x, y), x, y \in S$ is defined as $R_{i}^{-1}(x, y)=R_{i}(y, x)$. We use the prefix notation $R_{i}(x, y)$ for fuzzy relations, rather than the infix notation $x R_{i} y$, since the former is considered to be more convenient for the reader. The intersection, union and sup-t composition of any two fuzzy relations $R_{1}$ and $R_{2}$ defined on the same set of concepts $S$ are given by:

$$
\begin{gathered}
\left(R_{1} \cap R_{2}\right)(x, y)=t\left(R_{1}(x, y), R_{2}(x, y)\right) \\
\left(R_{1} \cup R_{2}\right)(x, y)=u\left(R_{1}(x, y), R_{2}(x, y)\right) \\
\left(R_{1} \circ R_{2}\right)(x, y)=\sup _{w \in S} t\left(R_{1}(x, w), R_{2}(w, y)\right)
\end{gathered}
$$

where $t$ and $u$ are a fuzzy $t$-norm and a fuzzy $t$-conorm, respectively. The standard $t$-norm and $t$-conorm are the min and $\max$ functions, respectively, but others may be used if appropriate. The operation of the union of fuzzy relations can be generalized to $M$ relations. If $R_{1}, R_{2}, \ldots, R_{M}$ are fuzzy relations in $S \times S$ then their union $R^{u}$ is a relation defined in $S \times S$ such that for all $(x, y) \in S \times S, R^{u}(x, y)=u\left(R_{i}(x, y)\right)$. A transitive closure of a relation $R_{i}$ is the smallest transitive relation that contains the original relation and has the fewest possible members. In general, the closure of a relation is the smallest extension of the relation that has a certain specific property such as the reflexivity, symmetry or transitivity, as the latter are defined in [16]. The sup- $t$ transitive closure $\operatorname{Tr}^{t}\left(R_{i}\right)$ of a fuzzy relation $R_{i}$ is formally given by:

$$
\operatorname{Tr}^{t}\left(R_{i}\right)=\bigcup_{j=1}^{\infty} R_{i}^{(j)}
$$

where $R_{i}^{(j)}=R_{i} \circ R_{i}^{(j-1)}$ and $R_{i}^{(1)}=R_{i}$. It is proved that if $R_{i}$ is reflexive, then its transitive closure is given by $\operatorname{Tr}^{t}\left(R_{i}\right)=$ $R_{i}^{(n-1)}$, where $n=|S|[16]$.

Based on the relations $R_{i}$ we first construct the following combined relation $T$ utilized in the definition of the taxonomic context $C$ :

$$
T=\operatorname{Tr}^{t}\left(\cup_{i} R_{i}^{p_{i}}\right), \quad p_{i} \in\{-1,0,1\}, \quad i=1 \ldots M
$$

where the value of $p_{i}$ is determined by the semantics of each relation $R_{i}$ used in the construction of $T$. More specifically:

- $p_{i}=1$, if the semantics of $R_{i}$ imply it should be considered as is

- $\quad p_{i}=-1$, if the semantics of $R_{i}$ imply its inverse should be considered

- $p_{i}=0$, if the semantics of $R_{i}$ do not allow its participation in the construction of the combined relation $T$.
The transitive closure in equation (9) is required in order for $T$ to be taxonomic, as the union of transitive relations is not necessarily transitive, independently of the fuzzy $t$-conorm used. In the above context, a fuzzy semantic relation defines, for each element $s \in S$, the fuzzy set of its ancestors and its descendants. For instance, if our knowledge states that "American Civil War" is before "WWI" and "WWI" is before "WWII", it is not certain that it also states that "American Civil War" is before "WWII". A transitive closure would correct this inconsistency. Similarly, by performing the respective closures on relations that correlate pair of concepts of the same set, we enforce their consistency.

Similarly, based on a different subset of relations $R_{i}$, we construct the combined relation $\widehat{T}$ for use in the determination of the runtime context $\widehat{C}$ :

$$
\widehat{T}=\cup_{i}\left(R_{i}^{\widehat{p}_{i}}\right), \quad \widehat{p}_{i} \in\{0,1\}, \quad i=1 \ldots \widehat{M}
$$

For the purpose of analyzing textual descriptions, relation $T$ has been generated with the use of a small set of fuzzy taxonomic relations, whose semantics are derived primarily both from the MPEG-7 standard and specific user requirements and are summarized in Table I. On the other hand, relation $\widehat{T}$ has been constructed with the use of the entire set of relations available in the knowledge base. This approach is ideal for the user modeling interpretation followed herein; initially, when dealing with generic user information, focus is given on the semantics of high level abstract concepts, whereas additional precision and a more specific view is required as the runtime user modeling expansion comes into play. The latter demands the use of all available information in the KB. Of course, as the construction of relation $\widehat{T}$ implies, an intermediate step of removing its possible cycles, that are present due to the utilization of all relations and their inverses, is necessary before the application of the taxonomy-based expansion process.

TABLE I. SEMANTIC RELATIONS USED FOR GENERATION OF COMBINED RELATION $T$.

\begin{tabular}{llllr}
\hline \multirow{2}{*}{ Name } & \multirow{2}{*}{ Inverse } & \multirow{2}{*}{ Symbol } & Meaning & \multicolumn{2}{c}{ Example } \\
\cline { 4 - 5 } & & & \multicolumn{1}{c}{$b$} \\
\hline Belongs & Owns & $\operatorname{Bel}(a, b)$ & $b$ belongs to $a$ & house device \\
Manufactured by & Constructs & $\operatorname{Made}(a, b)$ & $b$ is manufactured by $a$ & Siemens fridge \\
Friend & NotRelated & $\operatorname{Fr}(a, b)$ & $b$ is a friend of $a$ & George Bruno \\
Execute & ExecutedBy & $\operatorname{Exec}(a, b)$ & $b$ executes $a$ or & recipe user \\
& & & $a$ undergoes the action of $b$ & recipe rule \\
Triggers & TriggeredBy & $\operatorname{Trig}(a, b)$ & $b$ triggers $a$ &
\end{tabular}

The aforementioned relations are traditionally defined as crisp relations. However, in this work we consider them to be fuzzy, where fuzziness has the following meaning: high values of $\operatorname{Bel}(a, b)$, for instance, imply that the meaning of $b$ approaches the meaning of $a$, while as $\operatorname{Bel}(a, b)$ decreases, the meaning of $b$ becomes narrower than the meaning of $a$. A similar meaning is given to fuzziness of the rest semantic relations of Table I, as well. Based on the fuzzy roles and semantic interpretations of $R_{i}$, it is easy to see that both aforementioned relations (9) and (10), combine them in a straightforward and meaningful way, utilizing inverse functionality where it is semantically appropriate. More specifically, relation $T$ utilizes 
the following subset of relations:

$$
T=\operatorname{Tr}^{t}\left(B e l \cup M a d e^{-1} \cup F r \cup \text { Exec } \cup \text { Trig }^{-1}\right)
$$

Relation $T$ is of great importance, as it allows us to define, extract and use contextual aspects of a set of concepts. All relations used for its generation are partial taxonomic relations, thus abandoning properties like synonymity. Still, this does not entail that their union is also antisymmetric. Quite the contrary, $T$ may vary from being a partial taxonomic to being an equivalence relation. This is an important observation, as true semantic relations also fit in this range (total symmetricity, as well as total antisymmetricity often have to be abandoned when modeling real-life relationships). Still, the taxonomic assumption and the semantics of the used individual relations, as well as our experiments, indicate that $T$ is "almost" antisymmetric and we may refer to it as ("almost") taxonomic. Relying on its semantics, one may define the crisp taxonomic context $C^{\prime}$ of a single concept $s \in S$ as the set of its antecedents provided by relation $T$ in the ontology. Considering the semantics of the $T$ relation, it is easy to realize that when the concepts in a set are highly related to a common meaning, the context will have high degrees of membership for the concepts that represent this common meaning. Understanding the great importance of latter observation, we plan to integrate such contextual aspects of user models in our future work.

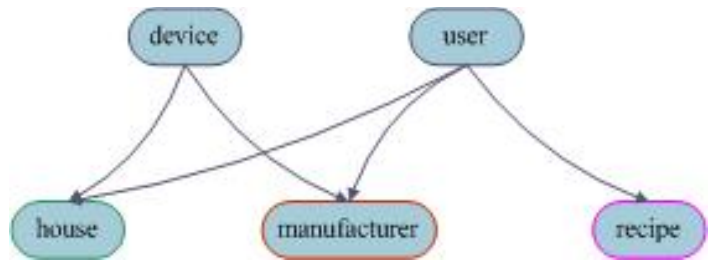

Fig. 1. Concepts and relations example.

As observed in Figure 1, concepts device and user are the antecedents of concepts house and manufacturer in relation $T$, whereas concept user is the only antecedent of concept recipe.

\section{SEMANTIC INTEROPERABILITy}

Another semantics related problem addressed within the SandS framework is connecting bidirectionally and ensuring interoperability between two formalization approaches with very different levels of semantic content, abstraction and granularity.

On one end, at the higher level of the SandS architecture, we have recipes for common household tasks produced and exchanged in the SandS Social Network that are described in near natural language (and the associated higher order semantics). A simplified XML recipe for washing clothes, routed to user defined by the UserID tag, is composed of three higher level instructions expressed in natural language inside the parameter $<$ R_Param $>$ tags, "Charge liquid", "Rotation velocity" and "Charge liquid", with their corresponding timing directives inside the $<$ R_Time $>$ tags, with either absolute values or " -1 " meaning after completion of the previous instruction and the actual values of the parameters ("on", "mean" and "off"). The reader is prompted to observe that the values can be words of a language with higher level semantics (for example the word "mean") with fuzzy meanings and interpretations.

On the other end, we have the domestic context of each user, which from the appliances' point of view consists of the actual appliances that the user has in house, each being of a certain type, model and brand. The SandS domestic middleware will be able through the UserID tag to associate immediately to a recipe for execution the exact domestic context of the user. Each appliance is ruled internally by an appliance-specific language that comprises the appliance's instruction set. Each instruction corresponds to a variable which has a semantic content restricted to exactly one operation of the machine and a set of associated acceptable values or parameter ranges for the operation. An example of two such variables for the aforementioned washing machine is shown in Figure 2.

\begin{tabular}{|c|l|l|}
\hline \multicolumn{3}{|c|}{ WASHING_MACHINE_SET_MOTOR_SPIN } \\
\hline Value & State & Description \\
\hline $0-200$ & WASHING_MACHINE_MOTOR_SPIN_VALUE & x10 rpm \\
\hline 255 & WASHIING_MACHINE_MOTOR_SPIN_REFRESH & Refresh \\
\hline
\end{tabular}

\begin{tabular}{|c|l|l|}
\hline \multicolumn{3}{|c|}{ WASHING_MACHINE_SET_WATER } \\
\hline Value & State & Description \\
\hline 0 & WASHING_MACHINE_WATER_STOP & Water stop \\
\hline 1 & WASHING_MACHINE_WATER_LOAD & Load water \\
\hline 2 & WASHING_MACHINE_SOAP_LOAD & Load soap \\
\hline 3 & WASHING_MACHINE_WATER_DRAIN & Drain water \\
\hline 255 & WASHING_MACHINE_WATER_STATUS_REFRESH & Refresh \\
\hline
\end{tabular}

Fig. 2. Example of two appliance-specific instructions. Semantics are strictly confined to variable-value pairs

Furthermore, it must be noted that the instruction set language is not unified even for the same type of appliances, since it is dependent to the manufacturer. These leads to an even bigger fragmentation of the semantic content and to a high granularity since specialized and unique to the manufacturer parameters and value sets are used to implement the actions that are necessary for the execution of the recipes that were described using almost natural language.

The proposed approach has similarities with ontology based context modeling approaches [17] which consider context as a specific kind of knowledge. This knowledge takes the form of mappings from recipe instructions to variable names and values that will be provided by experts which are the appliance manufacturers. An example of such a mapping is given in Figure 3.

Expert knowledge is needed to perform the variable mappings from the ontology describing the household tasks (recipe language) to the appliance capabilities ontology (instruction language) but it is also needed for assigning the right values to the variables. Note for example how two different actions, charge soap and drain water, correspond the same variable "WASHING_MACHINE_SET_WATER" but with different values ( 2 and $\overline{3}$ respectively) and also note how the naturallanguage-like "rotate in mean velocity" in the recipe is translated in WASHING_MACHINE_SET_MOTOR_SPIN=133, which in turn, from Figure 2, means spinning with a velocity of 1330 r.p.m. (assuming a maximum spin velocity of 2000 r.p.m. 


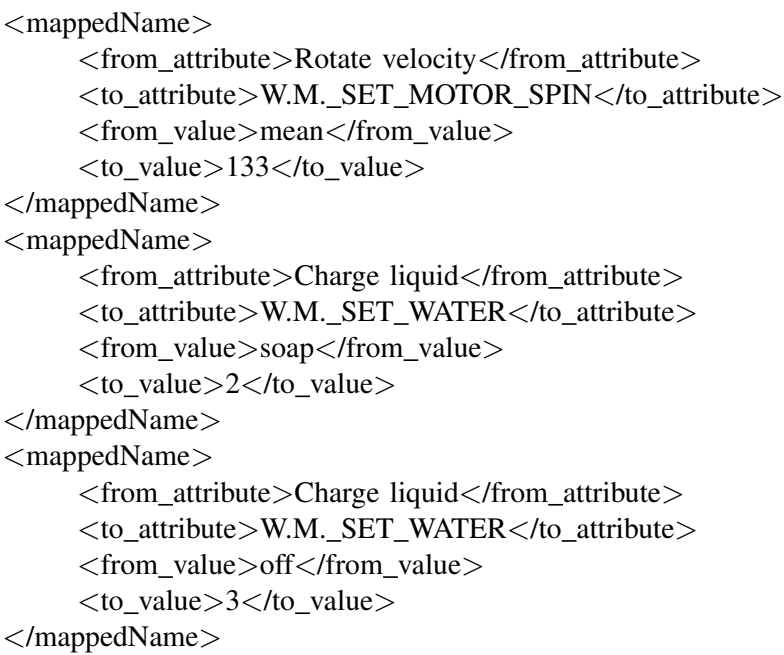

Fig. 3. Example of recipe's parameters and values to instruction's variables and values mapping. W.M. is an abbreviation for WASHING_MACHINE

and three steps, "low" at 660, "mean" at 1330 and "max" at 2000 r.p.m.).

The main advantage of this approach is that if there exist predefined mappings for all the appliances (including different types, models and brands) then the individual user and hence the entire SandS ecosystem could operate uniformly at the higher level using the language of the recipes which is too abstract semantically for machines but far more natural for humans. In this way it would be possible to accomplish household tasks without having to consider the specificities of the various appliances. Of course this will require the mappings to be made by the experts, but this could hardly be considered a workload problem since the mapping is only done once and could simply be added as an annex to the technical sheets already provided for the appliance. On the contrary, the challenging part for SandS partners, both academic and industrial, is to define the recipe language in such a way that it has enough semantic power to express all the possible appliance operations needed for the tasks but in the same time to produce meaningful mappings with the real hardware capabilities of the appliances.

An additional function of the overall pervasive architecture is its context awareness by performing home rule conformation check, and adaptation if needed, is performed. This rule conformation check is performed at the domestic level where home environment sensors constitute the input parameters of these rules and implement the domestic context awareness aspect of the SandS architecture. Such sensors include autonomous sensors (clock, luminosity, etc.), environmental and consumption sensors (electrical power consumption, humidity, rain, temperature) and higher level sensors such as the ones envisioned in the Smart City notion (municipality services). Actual rules for the domestic, or even geographical clusters of households, consist of a input/action pair. Input has been discussed as sensor providing domestic context information while actions consists of a more ad hoc user or system directive. For example for the home rule "If the weather is humid double the drying time of all washing devises" the input is provided by the humidity sensor and the action consists of adapting the drying time.

\section{EXPERIMENTAL VALIDATION PLAN}

At present, data for experimental validation of the proposed approaches is not yet available since SandS is still at it's early development stage. For this reason we researched bibliography mostly in the areas of pervasive computing and Smart Homes in order to detect datasets close to the requirements set during the design of the user and context modeling process described in the current article. Our research yielded some results that although relevant could not be placed within the scope of our research goals and could not meet the set requirements. These results include:

- Home Activity and Sensors Datasets [18] that were collected and reported also related to the CHI 2009 Workshop on Developing Shared Home Behavior Datasets to Advance HCI and Ubiquitous Computing Research. This collection of home activity datasets includes mainly instrumented living environments recorded data that are somewhat irrelevant to our research aims since we are not dealing with in house user behavior.

- the Ambient Intelligence Datasets [19] which contains links to smart home datasets, as well as data gathered from wearable sensors.

- the Smart* Data Set [20] that deals with energy consumption and continuously gathers a wide variety of data in three real homes, including electrical (usage and generation), environmental (e.g., temperature and humidity), and operational (e.g., wall switch events).

- the HomeData [21] which is a collection of publicly available datasets recorded from different homes for use in research on Load Disaggregation, Smart Homes, and Ambient Assisted Living.

- a common repository [22] for context recognition data sets initiated during Pervasive 2004 Workshop on Benchmarks and a Database for Context Recognition

- the ContextPhone [23] dataset on mobile context and communication.

- Nodobo [24] containing data gathered during a study of the mobile phone usage of high-school students, from September 2010 to February 2011.

Some first results on semantic interoperability will be available when an early release of the SandS middleware will be implemented. Experimental validation of the user modeling, formalization architecture and corresponding adaptation mechanisms would be possible when user, context and usage data could be collected from early to final entire Sands implementations. A small scale mockup that will replicate the overall SandS system will be composed of a physical site located in Cartif [25] and seven virtual sites each one located in a server for each partner. Data from the small scale mockup will be available on September 2014. Large scale validation of the SandS system will include deploying the 
system at the Crew [26], OpenLab [27] and SmartSantander [28] FIRE facilities to stress different aspects of applicability. Analysis of the collected data in terms of stereotype personas and usage/context analysis is required in order to construct the knowledge base and fuzzify the ontology definition. This analysis will be followed by integration of its results to the adaptation mechanisms of the SandS system. Large scale validation will end on October 2014 and will consist the main data source for these processes.

\section{CONCLUSIONS AND FUTURE WORK}

In this paper we illustrated how the emerging semantics of the Smart Home environments can be captured through a novel formalism and how expert knowledge can be used to ensure semantic interoperability. User stereotypes or personas on the one hand provide flexibility, extensibility, reusability and applicability and on the other hand knowledge management is incorporated as an efficient user and context model representation formalism. In addition, this formal, machineprocessable representation is used in order to define, extract and use both a set of concepts and their fuzzy semantic relations. Finally, it is demonstrated how expert knowledge can be used in the form of mappings to bridge the semantic gap between the natural language content of recipes, the main medium circulating inside SandS social network, and the semantically restricted set of instructions of the appliances in the user's domestic context.

Ongoing, the progress relying heavily on the issues raised in section $\mathrm{V}$, and future work includes incorporation of user, usage and context information, through a unified semantic representation, driving an adaptation mechanism aiming to provide a personalized service and optimizing the user experience. Among the aspects of the architecture that requires experimental validation is the computational cost and the scaling of SandS to a wider user group. Based on the SandS architecture the cloud infrastructure ensures the optimal handling of the computational load since the intermediate processes are not computationally demanding. On the other hand, issues that may arise from the scaling of the platform application are part of the experimental validation since the load is directly correlated with the user activity. The large scale validation will provide us with useful insights about the latter.

\section{ACKNOWLEDGMENTS}

Current research work has been funded by FP7, Future Internet Research and Experimentation - FIRE project Social \& Smart, Social housekeeping through intercommunicating appliances and shared recipes merged in a pervasive webservices infrastructure.

\section{REFERENCES}

[1] [Online]. Available: http://www.sandsproject.eu

[2] A. Kobsa, "Generic user modeling systems," User modeling and useradapted interaction, vol. 11, no. 1-2, pp. 49-63, 2001.

[3] P. T. A. Junior and L. V. L. Filgueiras, "User modeling with personas," in Proceedings of the 2005 Latin American conference on Humancomputer interaction. ACM, 2005, pp. 277-282.
[4] M. Baldauf, S. Dustdar, and F. Rosenberg, "A survey on context-aware systems," International Journal of Ad Hoc and Ubiquitous Computing, vol. 2, no. 4, pp. 263-277, 2007.

[5] C. Bettini, O. Brdiczka, K. Henricksen, J. Indulska, D. Nicklas, A. Ranganathan, and D. Riboni, "A survey of context modelling and reasoning techniques," Pervasive and Mobile Computing, vol. 6, no. 2, pp. 161180, 2010.

[6] P. Castells, M. Fernandez, and D. Vallet, "An adaptation of the vectorspace model for ontology-based information retrieval," Knowledge and Data Engineering, IEEE Transactions on, vol. 19, no. 2, pp. 261-272, 2007.

[7] S. Gauch, J. Chaffee, and A. Pretschner, "Ontology-based personalized search and browsing," Web Intelligence and Agent Systems, vol. 1, no. 3, pp. 219-234, 2003.

[8] A. Kiryakov, B. Popov, I. Terziev, D. Manov, and D. Ognyanoff, "Semantic annotation, indexing, and retrieval," Web Semantics: Science, Services and Agents on the World Wide Web, vol. 2, no. 1, 2011.

[9] B. Popov, A. Kiryakov, D. Ognyanoff, D. Manov, and A. Kirilov, "Kima semantic platform for information extraction and retrieval," Natural language engineering, vol. 10, no. 3-4, pp. 375-392, 2004.

[10] P. Castells, M. Fernández, D. Vallet, P. Mylonas, and Y. Avrithis, "Self-tuning personalized information retrieval in an ontology-based framework," in On the Move to Meaningful Internet Systems 2005: OTM 2005 Workshops. Springer, 2005, pp. 977-986.

[11] F. Baader, The description logic handbook: theory, implementation, and applications. Cambridge university press, 2003.

[12] T. R. Gruber et al., "A translation approach to portable ontology specifications," Knowledge acquisition, vol. 5, no. 2, pp. 199-220, 1993.

[13] D. L. McGuinness, F. Van Harmelen et al., "Owl web ontology language overview," W3C recommendation, vol. 10, no. 2004-03, p. 10, 2004.

[14] S. Miyamoto, "Fuzzy sets in information retrieval and cluster analysis," Theory and Decision Library, Ser. D: System Theory, Knowledge Engineering and Problem Solving, Dordrecht: Kluwer, 1990, vol. 1, 1990.

[15] S. Calegari and D. Ciucci, "Fuzzy ontology, fuzzy description logics and fuzzy-owl," in Applications of Fuzzy Sets Theory. Springer, 2007, pp. $118-126$.

[16] G. J. Klir and B. Yuan, Fuzzy sets and fuzzy logic. Prentice Hall New Jersey, 1995.

[17] X. H. Wang, D. Q. Zhang, T. Gu, and H. K. Pung, "Ontology based context modeling and reasoning using owl," in Pervasive Computing and Communications Workshops, 2004. Proceedings of the Second IEEE Annual Conference on. IEEE, 2004, pp. 18-22.

[18] [Online]. Available: http://boxlab.wikispaces.com/List+of+Home+ Datasets

[19] [Online]. Available: http://www.cise.ufl.edu/ prashidi/Datasets/ ambientIntelligence.html

[20] [Online]. Available: http://traces.cs.umass.edu/index.php/Smart/Smart

[21] [Online]. Available: https://github.com/smakonin/HomeData

[22] [Online]. Available: http://www.pervasive.jku.at/Research/Context_ Database/

[23] [Online]. Available: http://www.cs.helsinki.fi/group/context/\#data

[24] [Online]. Available: http://nodobo.com/release.html

[25] [Online]. Available: http://www.cartif.com/en/

[26] [Online]. Available: http://www.crew-project.eu/

[27] [Online]. Available: http://www.ict-openlab.eu/

[28] [Online]. Available: http://www.smartsantander.eu/ 